\title{
De quoi sont faits les faits grâce auxquels on parle d'histoires en Afrique noire ou ailleurs?
}

\author{
Alain Marliac \\ Directeur de recherches honoraire de l'IRD, en anthropologie et archéologie, 18 rue la Vieuville, 75018 Paris, France
}

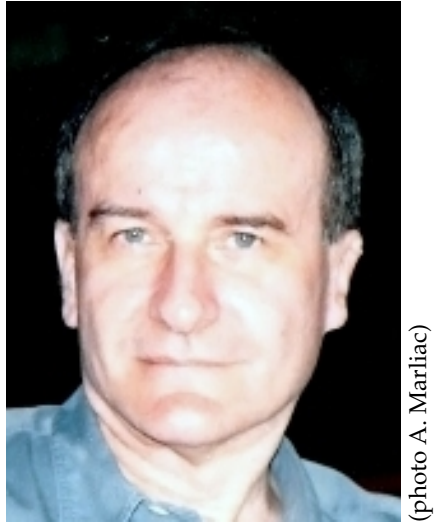

Ce que nous voulons savoir c'est comment un point de vue, un objet technique, finissent par s'imposer.

Callon, M., Latour, B., 1991.

La science telle qu'elle se fait,

Paris, La Découverte, 25.

À partir de mon expérience de terrain en Afrique subsaharienne des périodes préhistoriques à la lisière de l'histoire - étudiées aussi par les ethnohistoriens, linguistes comparatistes et historiens des premiers documents écrits $(\text { tarikh })^{1}-$, je tente ici de poser une question ouverte

\section{Auteur correspondant : a.marliac@free.fr}

Cet article fait suite à une communication orale lors de l'atelier "État des lieux de l'archéologie en Afrique», RTP "Études Africaines » du CNRS. Rencontres des 29, 30 novembre et $1^{\text {er }}$ décembre 2006 (in Marliac, A., 2008. L'archéologie à travers champs, textes et situation, soumis).

1 e.g. Marliac, A., 2006a. De l'archéologie à l'histoire. La fabrication d'histoires en Afrique subsaharienne et au-delà, Paris, L'Harmattan; Marliac, A., 2006b. Archéologie du Diamaré au Cameroun Septentrional. Milieux et peuplements entre Mandara, Logone, Bénoué et Tchad concernant l'état des lieux intellectuels, scientifiques et politiques des savoirs lors de la fabrication d'histoires. Comment rattache-t-on les faits archéologiques aux faits historiques en vue d'une Histoire des peuplements locaux ou régionaux?

$\mathrm{Ou}$, en très bref : que se passe-t-il lorsque tous les concernés - du social scientist aux profanes - parlent, discutent et tranchent d'Histoire ou d'histoires? Que l'on mélange ce que Licoppe ${ }^{2}$ appelle experientia (expérience partagée par tous) avec experimentum (expérience singulière réservée à des initiés, e.g. l'expérience scientifique, l'expérience archéologique) ? On ne peut envisager effectivement de détacher l'histoire dite " scientifique", d'abord des chercheurs qui la bâtissent, et de l'histoire vécue et dite par les personnes concernées.

\section{Constat}

À mon sens, la situation évoquée ici correspond à ce que l'on appelle - dans le domaine scientifique - les controverses/discussions/et même conflits/quelquefois forums ${ }^{3}$, qui débouchent parfois sur un résultat politicosocial faisant assez souvent confiance aux experts. C'est dans ces situations que l'on découvre comment les

pendant les deux derniers millénaires. BAR International Series 1549, Oxford, Cambridge Monographs in African Archaeology, 67.

${ }^{2}$ Licoppe, Ch., 1996. La formation de la pratique scientifique, Paris, La Découverte.

${ }^{3}$ Dont on sait le pouvoir pédagogique sur les participants et le pouvoir révélateur sur les intéressés, à partir d'expériences déjà réalisées de par le monde (les forums hybrides, $c f$. in Callon, M., Lascoumes, P., Barthe, Y., 2001. Agir dans un monde incertain. Essai sur la démocratie technique, Paris, Seuil, 49-60). 
deux grandes délégations qui caractérisent et divisent le monde moderne ${ }^{4}$ fonctionnent et posent problème ${ }^{5}$ :

- l'une déléguant aux scientifiques la définition du savoir véritable face aux profanes ;

- l'autre déléguant aux politiques le pouvoir réel face à leurs électeurs (démocratie représentative).

Je me limiterai ici à une réflexion-proposition dans ma propre discipline quand ses résultats reviennent dans le macrocosme selon le schéma proposé par M. Callon et al. ${ }^{6}$.

Ma compétence disciplinaire de préhistorien - que j'accepte pleinement d'ailleurs - tendrait à me restreindre à parler de domaines tout à fait en dehors de ce problème et des préoccupations d'une grande partie des africanistes intéressés par l'Histoire et donc à me marginaliser (le domaine de la préhistoire). Ce serait une erreur, car si les préhistoriens n'ont affaire qu'à des choses (au sens d'objets matériels), ils définissent ces choses et les relations entre ces choses, grâce aux hypothèses/interprétations anthropologiques sur ces choses et leurs mises en relations. On sait, au-delà, que les faits qu'ils fabriquent ainsi, possèdent un poids sociopolitique redoutable quant aux problèmes de l'origine de $1^{\prime} H_{0 m m e}{ }^{7}$ et de l'origine des Africains en particulier ${ }^{8}$.

Les problèmes de l'utilisation/mélange/enchaînement/tricotage, légitimes ou pas, de différents savoirs - qu'il s'agisse de préhistoire ou de protohistoire - utilisés, relèvent de la problématique, auparavant citée, des controverses. On constate facilement que, dans ce même domaine de la préhistoire, les faits de la préhistoire (restreints à des domaines dits fondamentaux) sont utilisés dans des discours, émissions, déclarations et articles qui passionnent nombre de lecteurs bien au-delà des spécialistes. Savoir pourquoi et comment cette passion naît et demeure nous aiderait sûrement à réfléchir à la pertinence de nos produits, car ces utilisations par des profanes s'effectuent en dehors du territoire de légitimité des résultats archéologiques. Ceux-ci en outre sont caractérisés par le fait que les archéologues sont à distance plus ou moins grande dans le temps, l'espace et par la compétence des fabricants des vestiges ${ }^{9}$. Mais n'est-ce pas

\footnotetext{
4 Latour, B., 2004. Politiques de la nature, Paris, La Découverte, 90 ; Callon et al., 2001, op. cit.

5 Voir les déclarations publiques, anciennes ou récentes ( $c f$. discours introductif à la conférence de l'INRAP « Modernité de l'Archéologie » par son directeur le 23.11.06), condamnant en les choisissant (sur quel argument? de quel droit?) les «mauvaises utilisations » de La Science.

6 Callon et al., 2001, op. cit.

7 Cf. les déclarations dogmatiques du professeur M. Brunet au sujet de la théorie de l'évolution.

8 Il n'est pas besoin d'aller bien loin pour mesurer leur présence et leur importance.

9 Latour, B., 2006. Changer de société - Refaire de la sociologie,
} Paris, La Découverte, 115. la réalité même d'une forme de transfert? de partenariat? de coopération? et même d'une forme de connaissance très commune, bien plus étendue, variée et instable que les connaissances scientifiques-archéologiques ou parfois médiatiques?

Mon maître, André Leroi-Gourhan, aurait probablement d'ailleurs et ironiquement rappelé combien cette science, déclarée si souvent inutile ${ }^{10}$, y compris par des collègues historiens, anthropologues, sociologues ou autres, comporte d'utilisateurs, de lecteurs, de revues et d'amateurs (certains fort savants) qui étripent la terre - dans tous les recoins du monde - à la recherche des origines, comme d'autres fouillent et dissèquent, sur un divan ou dans des romans, leurs souvenirs, leurs rêves ou leurs discours, à la recherche d'eux-mêmes ${ }^{11}$. Une certaine forme d'archéologie passionne les publics de 7 à 77 ans !

Je rappellerai, pour la curiosité d'autres disciplines, que cette passion - que je ne condamne sûrement pas puisqu'elle m'a saisi comme d'autres - est historiquement et philosophiquement, plus particulièrement occidentale et, pour une grande partie, moderne ${ }^{12}$ quant à sa discipline de référence : l'anthropologie. Quant à la Constitution fondamentale de l'anthropologie : la « constitution moderne » définie par B. Latour, elle constitue la base à la fois des sciences et de La Science à travers les disciplines ${ }^{13}$. Ce n'est pas sans incidence sur les produits d'une de ces disciplines : l'archéologie.

Pour les périodes abordées par l'archéologie historique (plus particulièrement africaine pour moi), méthodes et techniques sont grosso modo les mêmes qu'en archéologie préhistorique, même si la plus grande richesse des vestiges trouvés (ajoutée aux traditions orales, aux premiers manuscrits, aux premiers journaux de voyage) et l'utilisation de techniques (e.g. les méthodes de datations absolues croisées, les photos aériennes ou satellitaires) autorisent des développements plus étendus et meilleurs qui ne sont pas d'ailleurs que scientifiques. Divers théoriciens hissent ces résultats au niveau d'explications que je qualifierais plutôt d'hypothèses ou de propositions plus ou moins solides, ce qui n'enlève rien à leur respectabilité, mais leur donne une place plus conforme à ce qu'ils sont, à leurs conditions de fabrication (artéfacts + théories socio-anthropologiques $\rightarrow$ faits).

\footnotetext{
10 Et parfois même simplement auxiliaire de l'Histoire sans que les historiens identifient le hiatus entre leurs résultats et les nôtres.

11 Il n'est que de constater le nombre de revues de qualité, superbement illustrées, dédiées à l'archéologie-préhistoire chez les libraires. Je ne parle pas bien sûr des archéologies-fictions, intéressantes elles-aussi mais sur un autre plan (le continent de $\mathrm{Mu}, \mathrm{l}^{\prime}$ Atlantide, etc.).

12 Marliac, A., 2007b. À propos des objets et des mots de l'Anthropologie. Anthropologie E Sociétés, 31, 3, 185-204.

13 Latour, B., 1991. Nous n'avons jamais été modernes, Paris, La
} Découverte. 
Leur entrée dans le macrocosme ${ }^{14}$ provoque leur acceptation, leur remaniement, redéfinition ou parfois rejet, comme aussi le retraitement des «théories » qui émanent des différents composants de ce macrocosme rassemblés en collectifs ${ }^{15}$ plus ou moins puissants, étendus et hétéroclites, alliés, indifférents ou ennemis. Et c'est au niveau de cette entrée - comme déjà je le suggérais plus haut à propos du problème des origines de $\mathrm{l}^{\prime}$ Homme -, que je pense qu'un débat doit s'ouvrir car c'est là que se posent les incertitudes générées par tout le questionnement non scientifique des intéressés face au questionnement de la discipline sur elle-même.

\section{Essai de description}

Lorsque nous définissons et affirmons des faits archéologiques, que fabriquons-nous à ce moment-là ? Que se fabrique-t-il à ces moments-là ? Pourquoi tel ou tel choix? Pouvons-nous en faire une analyse, une exploration, un état des lieux? Je n'avance - dans ce qui suit - rien d'autre qu'une réflexion personnelle diversement argumentée autour du passage des faits dits scientifiques que nous - archéologues - produisons, (associés, selon des modes à discuter, aux produits d'autres disciplines : 1'interdisciplinaire), à d'autres faits, à d'autres points de vue, dont celui des besoins et des intérêts.

Ce passage, on pourrait le caractériser par la traduction, la transformation ou association des matter of facts (faits) et des matter of concerns (valeurs), que cette traduction soit une opération plutôt sémiotique telle que la définit M. Callon ${ }^{16}$ ou autre, picturale même, telle que la décrit M. Serres ${ }^{17}{ }$ c $^{\prime}$ est-à-dire non limitée au langage ${ }^{18}$.

Cette traduction, jadis illégitime et dédaignée, même si toujours existante ${ }^{19}$, il est devenu nécessaire sinon inévitable de $1^{\prime}$ analyser ${ }^{20}$. Faits et valeurs (comme les pôles Nature/Culture de la « constitution moderne») sont désormais pris comme des aboutissements évolutifs de médiations et non comme des points de départ distincts. La double délégation citée auparavant est mise en cause.

Rappelons-nous à ce propos que dans les représentations de faits archéologiques entrent aussi bien des phrases, des calculs (histogrammes, courbes, tableaux...),

\footnotetext{
14 Callon et al., 2001, op. cit.

15 Latour, 1991, op. cit.
}

16 Callon, M., 1980. Struggles and negotiations to define what is problematic and what is not: le socio-logic of translation, in Knorr, K.D., Krohn, R., Whitley, R. (Eds), The social process of scientific investigation: sociology of sciences, vol. IV, Dordrecht, D. Reidel, 211.

17 Serres, M., 1982, Turner translates Carnot, in Harari J.V., Bell D.F. (Eds) Hermes : Litterature, Science, Philosophy, Baltimore, Johns Hopkins, 62.

18 Latour, 2004, op.cit., 125.

19 Latour, 2004, op. cit., 140-148.

${ }^{20}$ Callon et al., 2001, op. cit., 316-319, 326. des enchaînements, des plans, que des images depuis le «dessin scientifique» (conventionnel par exemple pour les outils lithiques), les reconstitutions peintes ou sculptées jusqu'aux photos et au langage propre du chercheur (les élèves d'A. Leroi-Gourhan reconnaissaient bien son style, lié à sa formation particulière, son empirisme $\mathrm{e}^{21} \mathrm{sa}$ compétence bidisciplinaire et son teilhardisme discret). Évoquons au passage les créations artistiques les plus variées depuis les anciennes illustrations (e.g. celles des premières éditions de la Guerre $d u$ Feu de J.H. Rosny), les bandes dessinées les plus diverses ${ }^{22}$ (et les plus ridicules), le dernier film d'Yves Coppens, les reconstitutions les plus grandioses (e.g. au site de Stonehenge) et les photographies aériennes curieuses comme en Bosnie. ${ }^{23}$

Si j'ai rappelé le nombre d'actants (acteurs humains et non humains) qui sont à l'œuvre dans la fabrication des faits, on se doit de rajouter aussitôt le chercheur - enfermé dans son paradigme (kuhnien ou autre) ${ }^{24}$. Doté de ses postulats, méthodes, techniques, son lexique, ses intérêts, valeurs, alliances, etc., toujours tenté de se considérer comme surplombant le processus de fabrication en cours, il devient alors, soit un être immatériel représentant la Vérité (c'est-à-dire Dieu), soit un être réel armé de théories socio-anthropologiques, mais dès lors tout à fait discutables comme toute théorie scientifique digne de ce nom, à moins de sortir du domaine des sciences. Il s'agit alors de ces discours variés que l'on trouve souvent dans la grande presse maladroite ou intellectuellement formatée ${ }^{25}$.

Quand on parle d'Histoire ou d'histoires en Afrique noire et aussi en Europe (pour les périodes charnières comme la culture La Tène (450 av. J.-C. à 400 ap. J.-C. $\leftrightarrow$ peuples «celtes $»^{26}$ ), on gère ensemble - dans les proportions différentes selon les points de vue et les intérêts des objets scientifiques venant de l'archéologie, de l'ethnologie, de l'histoire (mettant de côté pour le moment la linguistique et l'anthropologie physique et d'autres disciplines ainsi que l'idéologie dominante). Ces objets scientifiques sont de tout ordre : poterie, métal, formes, décors, rites mortuaires, etc. avec datations, analyses physicochimiques en céramologie et en métallurgie, pédologie, géochimie, géomorphologie, flore, faune... Pour l'Afrique subsaharienne où la préhistoire finit tard après

\footnotetext{
${ }^{21}$ Latour, 2006, op. cit., 106

${ }^{22}$ Norbert, P., Liberatore, T., 2007. Lucy, Paris, Capitol Éditions. Postface Y. Coppens.

${ }^{23}$ Clichés où certains traits sont interprétés comme des pyramides (revue Nexus, 2006, No 45, 32-36).

${ }^{24}$ Le paradigme selon Kuhn. Cf. Kuhn, Th., 1972. La structure des révolutions scientifiques, Paris, Flammarion.

${ }^{25}$ Il n'existe pas de différence fondamentale quant aux postulats de leur vision du monde entre les journalistes de la « grande presse » et entre ceux des médias en général. Tous sont modernes.

${ }^{26}$ Leroi-Gourhan, A., 1988 (Ed). Dictionnaire de la Préhistoire, Paris, PUF, 868.
} 
le Christ, variablement selon les régions (vers la fin du XVIII ${ }^{e}$ au Diamaré, Cameroun du Nord) tout en maintenant ouverte la question de la scientificité des faits de ces disciplines et la question des traductions entre elles (pertes d'information, changements de référentiels, d'échelles), il faut désormais rajouter à ce court-bouillon - ce que l'on a toujours refusé de faire et que l'on continue de refuser (au nom du «progrès »/de la « démocratie ») les opinions publiques ou personnelles dictées elles aussi par l'intérêt dans tous les sens du terme y compris ceux plus ou moins clairs des chercheurs eux-mêmes et des institutions gouvernementales.

Si les « Histoires de l'Afrique », plus ou moins différentes exposées par le Pr. Martin Hall, le Pr. M’Bokolo, le président Wade, M. Obenga, M. Dika Akwa, le Pr. C.A. Diop $^{\dagger}$, le Pr. B. Lugan, le Pr. P. Breunig ou moi-même pour l'Afrique subsaharienne, ont quelque chose à voir les unes avec les autres, comment est-ce définissable? Comme elles ont aussi quelque chose à voir avec celles dites par les villageois ou citadins africains/européens, etc., et aussi avec celles dites par les autres disciplines, comment s'y retrouver? Nous sommes là en plein dans l'espace réel où se passent les activités de traduction, échelles, omissions, co-mensurations, positionnement, gestion et fabrication de faits, de nature tout à fait différente, pour des objectifs différents et éventuellement ennemis. Comment dans cette multiplicité plastique changeante, certains faits "durcissent-ils », e.g. deviennent-ils indiscutables et s'imposent-ils?

Comme exemple de «faits durcis », parlons de la conclusion - dans le temps et l'espace - de mes travaux quant aux civilisations de l'âge du fer au Cameroun du Nord, au sud de la plaine péritchadienne ${ }^{27}$, « région archéologique » précédemment totalement inconnue. C'est ce que je connais le mieux. Après tout un travail d'analyses puis de synthèses et comparaisons/juxtapositions, intégration de données environnementales et datations absolues, je conclus que deux cultures, le Salakien et le Mongossien, se partageaient la région considérée (le Diamaré) en deux phases culturelles contemporaines l'une de l'autre, entre le VII ${ }^{\mathrm{e}}$ siècle, le XIII ${ }^{\mathrm{e}}$ siècle et le XVIII ${ }^{\mathrm{e}}$ siècle de notre ère. L'une règne dans le Diamaré central, l'autre sur la limite du Diamaré et de la plaine du Logone plus au nord. Classiquement c'est l'établissement de typologies de poteries (morphologies, décors), la présence d'objets divers (fer, perles), de sépultures, répartis sur une stratigraphie archéologique (intrusions, sols d'occupation, fosses, états de surfaces), la répartition verticale des restes de faune et de flore plus la situation des datations absolues (TL et $\left.{ }^{14} \mathrm{C}\right)$ qui ont permis ces propositions.

Dans l'état actuel des recherches, connaissances, théories et instrumentations, ces propositions neuves sont l'état durci en «faits » d'artéfacts parfaitement discutables

\footnotetext{
27 Marliac, 2006b, op. cit.
}

et controversibles à tout moment par d'autres archéologues. D'abord dans leur base théorique (hypothèses anthropologiques), leur état d'aggrégats de connaissances variées (anthropologiques, pédologiques, climatologiques, palynologiques, physicochimiques et même tacites) puis par des recherches de même nature-éventuellement améliorées et étendues dans d'autres directions sur le même territoire. Ce fut le cas quand O. Langlois ${ }^{28}$ entreprit de travailler, dans la même région, sur des sites différents et similaires aux miens. Son étude diffère de la mienne dans la méthode, les techniques, les connaissances (e.g. importance donnée à la céramologie), typologie légèrement différente, calculs, etc., c'est-à-dire dans sa construction de «faits ». Ses conclusions diffèrent des miennes, entre autres, par la proposition que le Salakien se différencie aux alentours du XIII ${ }^{\mathrm{e}}$ siècle en post-Salakien. Ma conclusion générale ${ }^{29}$ tient compte de ses travaux à une certaine échelle ${ }^{30}$, puisque je propose une chronologie culturelle plus complexe, allant des premiers Postnéolithiques aux Postnéolithiques anciens, puis aux Postnéolithiques classiques (Salakien et Mongossien) pour finir par les Postnéolithiques nouveaux (post-Salakiens), introduisant donc une des propositions de mon collègue. Ceci pour conclure qu'il n'existe pas de « fait » définitivement établi mais des faits plus ou moins solidement durcis, compte tenu dans notre cas de l'immensité des recherches à entamer et à poursuivre.

Parallèlement, nous sommes encore dans l'ignorance des « retombées » dans le milieu de recherche archéohistorique camerounaise, dans l'enseignement, les médias, les publications vulgarisantes et le peuple camerounais dans ses diversités... J'ai tenté d'en faire une projection à petite échelle qui ne demande qu'à être améliorée ou rejetée par de futures recherches historiques, sociopsychologiques, linguistiques, etc. ${ }^{31}$. Aucun programme de collecte et analyse des connaissances archéologiques acquises n'a jamais été prévu, à ma connaissance, et ne le sera avant nombre d'années pour des raisons socioéconomiques et politiques assez évidentes. Je peux considérer ainsi nos résultats ${ }^{32}$ comme des «faits durcis », mis à part le principe, interne à toute discipline, que ces faits seront forcément « dépassés » un jour.

\footnotetext{
${ }^{28}$ Langlois, O., 1995. Histoire du peuplement postnéolithique du Diamaré (Cameroun Septentrional), thèse, université Paris 1 Panthéon-Sorbonne.

${ }^{29}$ Marliac, 2006b, op. cit.

${ }^{30}$ Depuis lors, hélas, aucune reprise des recherches archéologiques ne s'est déclenchée suite au désengagement de l'ORSTOM-IRD.

31 Marliac, A., 2005b. Archéologie et actualité dans l'ExtrêmeNord camerounais. Africa (Rome), LX, 3-4, 444-473.

32 Langlois, 1995, op. cit. ; Marliac, 2005c, Scientific discourses and local discourses: the case of African Archaeology, Intern. Journ. of Hist. Archaeol., 9, 1, 57-70.
} 
Il y a donc durcissement relatif des faits par absence/arrêt de controverse à leur sujet dans le domaine archéologique même s'il peut y avoir, dans le même temps, controverse dans le domaine anthropologique quant à la réalité de ces faits par rapport aux faits collectés par l'anthropologie culturelle concernant les peuples actuels régionaux traditionnels supposés «descendre » de ces cultures. Ainsi I. de Garine ${ }^{33}$ déclare, sans avoir fait de fouilles véritables, que les témoins culturels anciens/traditionnels collectés (comment?) au milieu des ethnies qu'il a étudiées (Massa, Mousgoum, Toupouri, Moussey) font preuve d'une remarquable similitude sauf à pratiquer - dirais-je - une analyse plus fine des objets pour la définition de nouveaux « faits ». Moi-même dans la ligne de réflexion présentée ici, j'avais déjà souligné l'inadéquation entre nos « unités » et les « unités » définies par l'ethnohistoire locale ${ }^{34}$.Une controverse anthropologicohistorique de ce type peut avoir des répercussions sociopolitiques, ou naîtra elle-même de controverses sociopolitiques si quelqu'un s'interroge quant à la nature des ethnies traditionnelles constitutives des ethnies actuelles et de la nationalité camerounaise d'aujourd'hui et à venir.

Ainsi, il n'existe pas de parenté morphologique et décorative nette entre les cultures matérielles traditionnelles (poteries surtout) les plus anciennes dans la tradition, et les typologies construites pour le Salakien et le Mongossien. Par conséquent, soit la divergence des faits prouverait l'absence de filiation soit la filiation se fait ailleurs, sachant que les typologies sont des regroupements abstraits d'ensembles de traits toujours changeants, soit encore les ethnies sont des entités toujours en évolution. Certains ethnologues et historiens modernes que la «constitution moderne » entraîne vers le constructivisme pousseraient jusqu'à dire qu'il n'y a pas d'ethnies ${ }^{35}$ ce que nous avons rejeté dans d'autres publications ${ }^{36}$ et que la triste réalité dément régulièrement de façon sanglante comme au Sri Lanka, au Kosovo ${ }^{37}$, au Kenya entre les Luo, Kalenjing et Kikuyu, après les Hutu et Tutsi au Rwanda.

\footnotetext{
33 de Garine, I., 1981. Contribution à l'histoire du mayo Danaye (Massa, Toupouri, Moussey, Mousgoum), in Tardits, C. (Ed.). Contribution de la recherche ethnologique à l'histoire des civilisations du Cameroun. Colloq. Internat. CNRS, 551, 1, 171-186.

${ }^{34}$ Marliac, A., 1995. Connaissances et savoirs pour l'Histoire : le cas du Nord-Cameroun. Africa, L, 3, 325-341; Marliac, A. 1997. Archaeology and Development: a difficult dialogue. Intern. Journ. Hist. Archaeol., I, 4, 330 ; Marliac 2006a, op. cit., 37.

35 Amselle, J.-L., M'Bokolo, E. (Eds) 1985. Au cœur de l'ethnie. Ethnies, tribalisme et état en Afrique, Paris, La Découverte.

36 Par exemple : Marliac, A., 2004. Scientific discourses plus local discourses: the future for Development issues? in Confronting XXIst Century Challenges, vol. I, Part 2, Makerere Univ. Printery, Kampala, Uganda ; Marliac, A., 2005a. From archaeological problems to development issues and beyond, Pratnatattva, 11, 65-74, Jahangirnagar Univ., Dhakka, Bangladesh.

37 Deuxième état de la planète reconnu sur une base monoethnique.
}

Cette constatation de changements n'implique pas en effet à l'échelle de quelques générations l'absence $\mathrm{d}^{\prime}$ appartenance ethnique ${ }^{38}$. Dans ce cas, la typologie des caractéristiques matérielles des cultures n'est pas pertinente quant à une éventuelle filiation. Deux cultures peuvent être reliées dans le temps sans forcément partager une culture matérielle identique mais, pour l'archéologue, la «culture matérielle» reste le matériau essentiel de ses typologies.

\section{Essai de découpage}

Ce que nous voudrions isoler - par curiosité intellectuelle et souci de savoir de quoi nous parlons - c'est, localement de préférence, l'espace compris entre le monde des opinions ordinaires, le monde des opinions de groupes et le monde dit des "faits scientifiques », monde où ces opinions, artéfacts et faits convergent et où éventuellement certains coagulent, durcissent, émergent, se chevauchent, se traduisent les uns dans les autres en se modifiant... Dans le monde moderne, ces activités et leurs produits sont de plus, transportés, surmultipliés, modifiés, tronqués, échangés, inversés ou simplement omis/ignorés (e.g. par l'enseignement et les média). De nos jours, certains durcissent en dogmes intouchables installant un régime de pensée généralisé et parfois légalisé, contradictoire et mortifère pour le monde de la recherche en sciences sociales.

Par principe, je n'en rejette préalablement aucune, mais je souligne que ces différents exposés/discours écrits, télévisés, médiatisés, reproduits ou pas, sont la réalité de l'archéologie, donc de l'archéologie historique en Afrique.

Il n'y a pas de faits archéologiques isolés, nus, non attachés ${ }^{39}$, une sorte d'archéologie située hors de la caverne platonicienne à laquelle les archéologues auraient, par extraordinaire, seuls accès ${ }^{40}$. L'archéologie se manifeste à différents moments et différents lieux sous différents lexiques, ouvrages, catalogues, différentes activités, représentations ou images plus ou moins solides, en référence à des visions du monde, des instrumentations, des situations, des pouvoirs et intérêts particuliers et des constitutions/cosmogonies variées ${ }^{41}$ plus ou moins organisés ou stratégiquement «alignés ». La vérité de l'archéologie,

\footnotetext{
38 Marliac, A., 2007a. in L'interdisciplinarité en question. Les choses, les mots et le passé des hommes, Paris, L'Harmattan, 149, $173,193$.

39 Callon et al. 2001, op. cit., 194.

${ }^{40}$ Latour, 2004, op. cit., chap. 1.

41 On peut ainsi rappeler les différences entre les diverses représentations de l'homme préhistorique qui ont jalonné l'histoire de la discipline depuis la statue des Eyzies-de-Tayac (illustrée dans Leroi-Gourhan, A., 1964. Le geste et la parole. Technique et Langage. 2 vol., Paris, Albin Michel, 38, fig. 5) jusqu'aux toutes dernières reconstitutions d'Homo sapiens, Homo neanderthalensis et Australopithecus afarensis (cf. ici, note 8).
} 
tout à fait transitoire, n'apparaît qu'en cas d'extinction d'une controverse dans son domaine. Cette controverse peut - très légitimement pour des scientifiques (ici les archéologues) - être relancée à tout moment sauf « lois mémorielles » dont on ignore quelle «nuit du 4 août » les attend.

À propos du passé, on peut concevoir ainsi qu'à tout instant au sein d'un groupe il existe toujours même si non verbalisée ou non figurée - une configuration/compréhension générale du monde composée de faits, d'artéfacts, de notions, de concepts plus ou moins ordonnés, plus ou moins solidifiés par différentes méthodes, dont la coercition et l'itération séculaire.

Cette configuration va d'un extrême abstrait indistinct (parallélisable métaphoriquement à la notion d'entropie en thermodynamique; like the state of total entropy ${ }^{42}$ ), à un autre extrême solidifiant diverses conceptions (dont les pensées dominantes que l'on ne peut nier et qui composent la cosmogonie/constitution/vision générale momentanée du monde occidental et ses extensions). Ces deux bornes laissent entre elles un espace conceptuel de sous-configurations en créations, conflits et associations constants, portant des définitions localisées/limitées/acceptées/contestées de choses, d'humains, d'artéfacts, aussi bien matériels qu'idéels mis et pliés ensemble (crumpled, comme dit Michel Serres ${ }^{43}$ ) et en compétition avec la configuration dominante.

Ces sous-figurations ou réseaux réussissent/échouent, perdurent ou pas, c'est-à-dire durcissent ou pas, longtemps ou pas. Leur milieu d'apparition est cet espace dont nous parlions plus haut, leurs conditions de durcissement sont le nombre et la qualité, la nature des alliés, qu'ils se trouvent et enrôlent, dans et hors les sciences.

Artéfacts et faits sont conçus, construits grâce à différents éléments selon des préconcepts, méthodes, techniques, associations, langages, bricolages, montages, proportions, selon aussi des rencontres, des pouvoirs, plus ou moins concurrents, etc., appuyés, inconsciemment/invisiblement pour le moment, sur la dichotomie Nature/Culture-social ${ }^{44}$ de la « constitution moderne ${ }^{45}$.

$\mathrm{Si}$, comme on dit trop banalement, « l'interrogation sur les origines est sans doute aussi ancienne que l'humanité ${ }^{46}$, si les hommes se sont toujours intéressés à leur passé, il est tout aussi évident que leurs approches du passé furent extrêmement diverses et évolutives en fonc-

\footnotetext{
${ }^{42}$ Brown, S.D., Capdevila, R., 2004. Perpetuum mobile: substance, force and the sociology of translation, in Law, J., Hassard, J. (Eds), Actor Network theory and after, Blackwell Publishing, 26-50.

43 Serres, 1982, op. cit.

44 Notions désormais réduites, pour ce qui me concerne, à des aboutissements et non déjà présentes avant toute prise en considération du problème.

45 Latour, 1991, op. cit.

46 Introduction à la conférence "Modernité de l'archéologie » INRAP, 23-24 novembre 2006.
}

tion de leurs cosmogonies (constitutions prémodernes), de leurs moyens de connaissance et de leur rapport au monde ${ }^{47}$.

Les configurations dont nous avons parlé sont susceptibles de varier dans l'espace comme dans le temps (ce dernier même allant du temps universel, aux temps locaux comme aux temps des narrations). En Europe déjà, il aura fallu différentes modifications de la cosmogonie des Européens à partir de la Renaissance, puis des premières avancées des sciences exactes grâce à « la révolution copernicienne », aux nouvelles instrumentations et différents bouleversements socioéconomiques laissant apparaître des alliés socioéconomiques/sociopolitiques pour les sciences, pour que naisse, à la fin du XIX ${ }^{\mathrm{e}}$ siècle, notre discipline, l'archéologie préhistorique, sous la « constitution moderne » qui nous régit toujours ${ }^{48}$.

Hors du monde Nord-Atlantique et de ses extensions, ce qui est notre situation professionnelle habituelle, les choses sont encore peu modifiées sauf par des intellectuels ayant acquis ce savoir occidental et la cosmogonie/constitution qui va avec ${ }^{49}$ plus tous les problèmes sociopsychologiques - et leurs conséquences politiques qui accompagnent cet apprentissage $\mathrm{e}^{50}$. Il suffit de lire les écrits et de suivre les parcours intello-sentimentaux de ces chercheurs/intellectuels africains ${ }^{51}$, qui résonnent en déphasage plutôt africanophile (sinon parfois raciste), face aux écrits repentants et parcours pénitents de tels intellectuels occidentaux.

En résumé et au niveau général, il se passe à chaque instant un brassage mettant en relations divers actants afin de définir un objet : pour nous - sous l'égide générale de l'anthropologie - telle ou telle définition par exemple d'une évolution, invention, modification, stase... à partir de la mise en réseau de différentes descriptions, en fonction de leurs significations, intérêts et valeurs.

En général, nos activités professionnelles se passent en amont de cette mise en réseau, tout en étant ellesmêmes des mises en réseau de descriptions, mesures, analyses, de nature très différentes. Par exemple pour un site donné : morphologie, sédimentologie, pédologie,

\footnotetext{
47 In Marliac, 2007a, op. cit., 121 ; Marliac, 2007b, op. cit.

48 Laming-Emperaire, 1963. L'Archéologie préhistorique, Paris, Le Seuil.

49 Diop, C.A., 1979. Nations nègres et culture. Paris, Présence Africaine; Obenga, Th., 2001. Le sens de la lutte contre l'Africanisme eurocentriste, Paris, L'Harmattan; Ela, J.-M., 2007. Recherche scientifique et crise de la rationalité, Paris, L'Harmattan, pour l'Afrique subsaharienne.

50 Marliac, A., 2001a. Problèmes archéologiques et problèmes humains : moi, nous et les autres. BAR International Series, 1522, Oxford, 153-161.

51 Diop, 1979, op. cit. ; Ki Zerbo, J., 1978. Histoire de l'Afrique noire, Paris, Hatier ; Dika Akwa, N.O., 1985. Les descendants des pharaons à travers l'Afrique, Paris, Osiris-Publisud ; Obenga, 2001, op.cit. ; Mudimbe, V.Y., 2000. Race, identity, politics and history. Journal of African History, 41, 291-294; Ela, 2007, op. cit.
} 
stratigraphie, paléozoologie, paléobotanique, interprétation anthropologique du site, de ses limites et troncatures, interprétation anthropologique des pièces lithiques, céramiques et autres (ossements, bois, cornes), ce que les archéologues français nomment très justement « l'anthropologie des techniques». Toute apparition (occurrence) dans un site est susceptible d'interprétations provenant des deux domaines que notre "constitution moderne" sépare ontologiquement : la Nature et la Culture ${ }^{52}$, les Faits et les Valeurs?

Mais, de plus, résultats obtenus et durcis (à la suite des traductions 1 et 2 de Callon et al. ${ }^{53}$ ) passent au moment de la traduction 3 (idem) dans l'ensemble des collectifs constituant ce que généralement on a appelé « la société ». Elle modifie et s'associe alors à un ou plusieurs d'entre eux et pas à d'autres. Elle et le chercheur qui l'a proposée se modifient eux-mêmes dans ce mouvement.

\section{Que chercher?}

Une fois débrouillés non pas tous les réseaux - ce qui me paraît un but théorique hors d'atteinte - mais certains particulièrement pertinents par rapport à la question posée, le problème restera de déterminer à quel moment tel réseau prime sur les autres, à quel moment, en quelque sorte, il démarre pour prendre une certaine consistance, durcir et perdurer. Ce sont en effet les réseaux qui "réussissent», "durent», plus ou moins longtemps dans l'espace et le temps, qui nous intéressent car ce sont ceux-là qui constituent, avec d'autres éventuellement, les croyances établies/les tendances lourdes/les politiques/la (ou les) dominance(s) idéologique(s) d'une époque ou encore l'innovation.

Depuis son installation en Europe sous sa forme moderne, La Science est la continuation de la politique par d'autres moyens (sans être réductible à cela) ; c'est-àdire que le détour par le laboratoire, lorsqu'il est réussi ou accepté, a pour conséquence, sinon comme projet, de reconfigurer les mondes dans lesquels nous décidons de vivre ${ }^{54}$. Ce détour (lui-même discutable ${ }^{55}$ ) n'existe pas en anthropologie dont se réclame l'archéologie, africaine ou pas. Le laboratoire est remplacé par le consensus établi des chercheurs ${ }^{56}$, consensus appuyé sur un collectif sociopolitique accepté par l'État qui finance la plupart d'entre nous. Et la façon dont les chercheurs intéressent la société dans laquelle ils vivent et travaillent est étroitement corrélée à la configuration sociale du moment en même temps qu'au type de pratique scientifique qu'ils développent ${ }^{57}$.

Pour conclure beaucoup trop vite sur le sujet, nous souhaiterions que nos pratiques, tout en s'améliorant (nouvelles techniques et méthodologies), cessent de suivre systématiquement les dominances sociopolitiques modernes actuelles déguisées sous le terme de consensus ${ }^{58}$, qui relève plutôt d'une collusion, visible au grand jour grâce au même vocabulaire et aux mêmes argumentaires, réitérés depuis deux siècles, recopiés de discours en éditoriaux, d'émissions en reportages, d'analyses dites «scientifiques », en dénonciations : c'est un collectif rassemblant les intérêts de la finance, l'industrie, telle politique, tels projets, tels futurs, etc.

Que nos pratiques prennent plutôt en compte les situations d'incertitudes (au plan théorique comme au plan pratique), les autres collectifs pour le moment sans pouvoir. «Il n'y a pas de manière "neutre" de définir le rôle des sciences dans nos sociétés [...] parce qu'il s'agit d'une question [...] qui engage l'ensemble de ses interprètes et qui les fait dérailler dès que ces interprètes, scientifiques inclus, revendiquent l'autorité des "matters of fact" » ${ }^{59} . \mathrm{Qu}^{\prime}$ elles ne contribuent pas à refermer ce souscollectif qu'est notre discipline sur des critères modernes et postmodernes, ni sur sa propre autosatisfaction quelle qu'elle soit, selon la formule "The more disconnected a discipline is from society, the better ", mais qu'elles s'engagent au-delà, par des moyens, à innover, à construire, à inventer, dans la composition avec les savoirs autres présents dans toute société afin que "The more connected a scientific discipline is to society, the better $»{ }^{60}$.

\footnotetext{
52 Marliac, A., 2001b. Du dialogue pédo-archéologique à un discours hybride? Com. Colloq. ICoTEM, Université de Poitiers, 11-12 octobre 2001, cité in Marliac, 2007a, op. cit. 121-147.

${ }^{53}$ Callon et al., 2001, op. cit., 75-104.
}

\footnotetext{
${ }^{54}$ Cf. Callon et al., 2001, op. cit., 101-102, citant Latour.

55 Callon et al., 2001, op. cit., 282.

56 Le consensus (entre les chercheurs et d'autres. A.M.) est souvent le masque qui cache les rapports de domination et d'exclusion (Callon et al., 2001, op. cit., 16).

57 Callon et al., 2001, op. cit., 93-94.

$58 \mathrm{Cf}$. note 56 .

59 Stengers, I., 2006. in La Vierge et le neutrino, Paris, Les Empêcheurs de penser en rond, 24.

60 Latour, B. 1998. From the world of science to the world of research? Science, 280, 209.
} 